\title{
Exploring potential chemical markers by metabolomics method for studying the processing mechanism of traditional Chinese medicine using RPLC-Q-TOF/MS: a case study of Radix Aconiti
}

\author{
Yubo $\mathrm{Li}^{1+}$, Yuming Wang ${ }^{1+}$, Lina Su${ }^{2+}$, Lixin $\mathrm{Li}^{1}$ and Yanjun Zhang ${ }^{1 *}$
}

\begin{abstract}
Background: Pao zhi is a common traditional approach that usually occurs before most herbs are prescribed whereby during processing, secondary plant metabolites are transformed, thus helping to increase potency, reduce toxicity and altering their effects. Using Radix Aconiti (Chuan Wu, CW) as a model herb, suitable chemical markers are crucial for studying the processing mechanisms of these herbs.

Results: In this study, the comprehensive metabolomic characters of CW and Prepared CW (ZCW) by RPLC-Q-TOF/MS were investigated to guarantee clinical safety. Multivariate analyses successfully identified specific metabolite changes between CW and ZCW. In addition, 22 key biomarkers responsible for the detoxifying actions of pao zhi were discovered. The processing mechanism of CW were discussed according to the identified metabolites. This method is efficient, providing more accurate characterisations of traditional pao zhi detoxification.

Conclusions: The proposed strategy proves that RPLC-Q-TOF/MS-based metabolomic analysis does not only explore chemical markers but can also provide a comprehensive understanding of the transformation mechanisms underlying pao zhi.

Keywords: Metabolomics, Radix Aconiti, Chuan Wu, Reversed phase liquid chromatography/quadrupole time-of-flight tandem mass spectrometry
\end{abstract}

\section{Background}

Radix Aconiti (Chuan Wu, CW) is the dried mother root of Aconitum carmichaeli Debx. This root is an essential drug in Traditional Chinese Medicine (TCM) and has been used for thousands of years. The herb is widely distributed in Sichuan Province (located in southwestern China), and has a wide range of pharmacological effects. Although CW has a limited therapeutic range, it is commonly used to treat various diseases such as collapse, syncope, rheumatic fever, painful joints, gastroenteritis, diarrhoea, oedema, bronchial asthma, and several tumors

\footnotetext{
*Correspondence: tianjin_tcm001@sina.com

${ }^{\dagger}$ Equal contributors

${ }^{1}$ Tianjin Key Laboratory of TCM Chemistry and Analysis, School of Traditional Chinese Materia Medica, Tianjin University of TCM, Tianjin 300193, P. R. China Full list of author information is available at the end of the article
}

[1-4]. Prepared CW (ZCW) is traditionally manufactured by boiling raw $\mathrm{CW}$ at $100^{\circ} \mathrm{C}$ for $8 \mathrm{~h}$ before drying it. More than 20 commonly used proprietary herbal products from both historical medical literature and modern clinical research reports contain $\mathrm{CW}$ or ZCW as main ingredient or auxiliary ingredient. These products include 'Wutou Tang,' 'Chuanfu Wan', 'Wufu Jiaojiang Tang', 'Zhentongning Injection' and 'Fengshigutong Jiaonang' etc.. In TCM, CW and ZCW have different uses and potential toxicity. CW is strongly toxic and is used externally; whereas ZCW has a 'warning toxicology', and is taken orally or injected. Therefore, consuming the wrong form of herb may lead to undesirable clinical outcomes. Hence, quality control of this herb is paramount. 
Pao zhi is a common approach that usually occurs before most herbs are prescribed whereby during processing. The role of pao zhi is to strengthen the effect, eliminate or reduce the toxicity, facilitate the preparation and storage of drugs. During processing, secondary plant metabolites are transformed, thus helping to increase potency and reduce toxicity, and altering their effects [4]. As a detoxifying measure, Paozhi is necessary to remove the poisonous Aconitum alkaloids mainly deriving from the diester diterpene alkaloids (DDAs) including aconitine, mesaconitine and hypaconitine [4]. They can be decomposed into less or non-toxic derivatives through Paozhi that plays an essential role in detoxification. The main mechanisms underlying herb processing were found to be mainly related to changes in composition and/or activity of herb components [5,6]. However, the difference in global metabolomic characters between CW and ZCW remains unclear. This difference restricts further application of ZCW in a clinical environment.

Metabolomics is a branch of science concerned with the total metabolome of integrated biological systems and dynamic responses to alterations of endogenous and/or exogenous factors [7]. The objective of 'nontargeted' metabolic profiling analysis is to detect as many metabolites as possible in a certain sample. Several papers have illustrated that metabolomics has been used in evaluating the pharmacological and toxicological effects of aconite products $[8,9]$. With the development of accurate, precision and new analytical techniques, metabolomics can provide global, comprehensive, detailed and reliable pieces of evidence for further studies and determination on efficacy/toxicity of CWs. Several methods have been developed for analysing aconitine alkaloids in CWs. These methods include high-performance liquid chromatography (HPLC), ultraviolet spectrophotometry (UV) and reversed phase liquid chromatography/quadrupole time-of-flight tandem mass spectrometry (RPLC-Q-TOF/ MS) [10-12]. However, research is still limited for content changes of several main alkaloids, while could not exploring potential chemical markers for studying the processing mechanism of $\mathrm{CW}$.

In this study, an approach that uses RPLC-Q-TOF/MS and pattern recognition analysis was developed to rapidly find potential chemical markers for studying the processing mechanism of Radix Aconiti. The protocol was executed using three steps. Firstly, this proposed strategy used RPLC-Q-TOF/MS to scan the full metabolic profiling of raw and processed Radix Aconiti. Secondly, a multivariate statistical analysis by principal component analysis (PCA) and partial least squares discriminant analysis (PLS-DA) of the mass spectrometry (MS) spectra based on all chemical information was conducted to find potential chemical markers. Lastly, the underlying regulations of pao zhi perturbed metabolic pathways were discussed and the processing mechanism of CW was elucidated according to the results of chemical markers for CW and ZCW. This novel method can be valuable for rapidly exploring potential chemical markers and studying processing mechanisms of herbs.

\section{Results and discussion}

\section{Acquisition and processing of metabolic profile data}

Global profiling of RPLC-Q-TOF/MS positive ion mode was analysed by RPLC-Q-TOF/MS to compare metabolic difference between $\mathrm{CW}$ and ZCW. As shown in Figure 1, low molecular mass metabolites have a good resolution and the baseline can be satisfactorily separated in $60 \mathrm{~min}$. PCA and PLS-DA were used to classify metabolic phenotypes and to identify different metabolites in order to evaluate variation among complex data sets. PCA is an unsupervised pattern recognition method without prior data set information. It retains maximum variance of multidimensional data whilst reducing their dimensionality. PCA score plots were obtained using metabolic data, an obvious separation trend can be observed between CW and ZCW samples in Figure 2A. The loading plot of supervised PLS-DA was used to investigate deeply differences between $\mathrm{CW}$ and $\mathrm{ZCW}$ and to find potential biomarkers. Figure $2 \mathrm{~B}$ displays the result of the PLS-DA model using metabolic data. A plot for variable importance parameters (VIPs) was used to identify metabolites according to the order of their contributions to clustering separation [4]. As shown in Table 1, mass spectrometry signals responsible for differentiation were characterised by the values of the VIPs (where a VIP value of $>1$ is regarded as significant) from the PLS-DA analysis [4]. According to the values of VIPs and the corresponding PLS-DA loading plots, 22 metabolites were identified and selected as potential biomarkers to distinguish striking difference of $\mathrm{CW}$ and $\mathrm{ZCW}$.

\section{Biomarker characterisation}

PCA results displayed as score plots allowed us to compare metabolite profiles of two different groups. PLS-DA loading plots analysis showed distinct metabolites clustered according to the characteristic change of raw or processed sample profiles $[4,8,9]$. Metabolite identifications were achieved by comparing retention time and MS data (accurate mass, isotopic distribution and fragmentation patterns in positive ion modes) of compounds with alkaloid compounds reported in literature and found in public online databases, or confirmed with standard compounds available in-house (Figure 3). Values for VIPs reflect the influence of each metabolite ion on classification. In addition, values for VIPs are calculated by the formula described in the user's guide of SIMCA-P. 


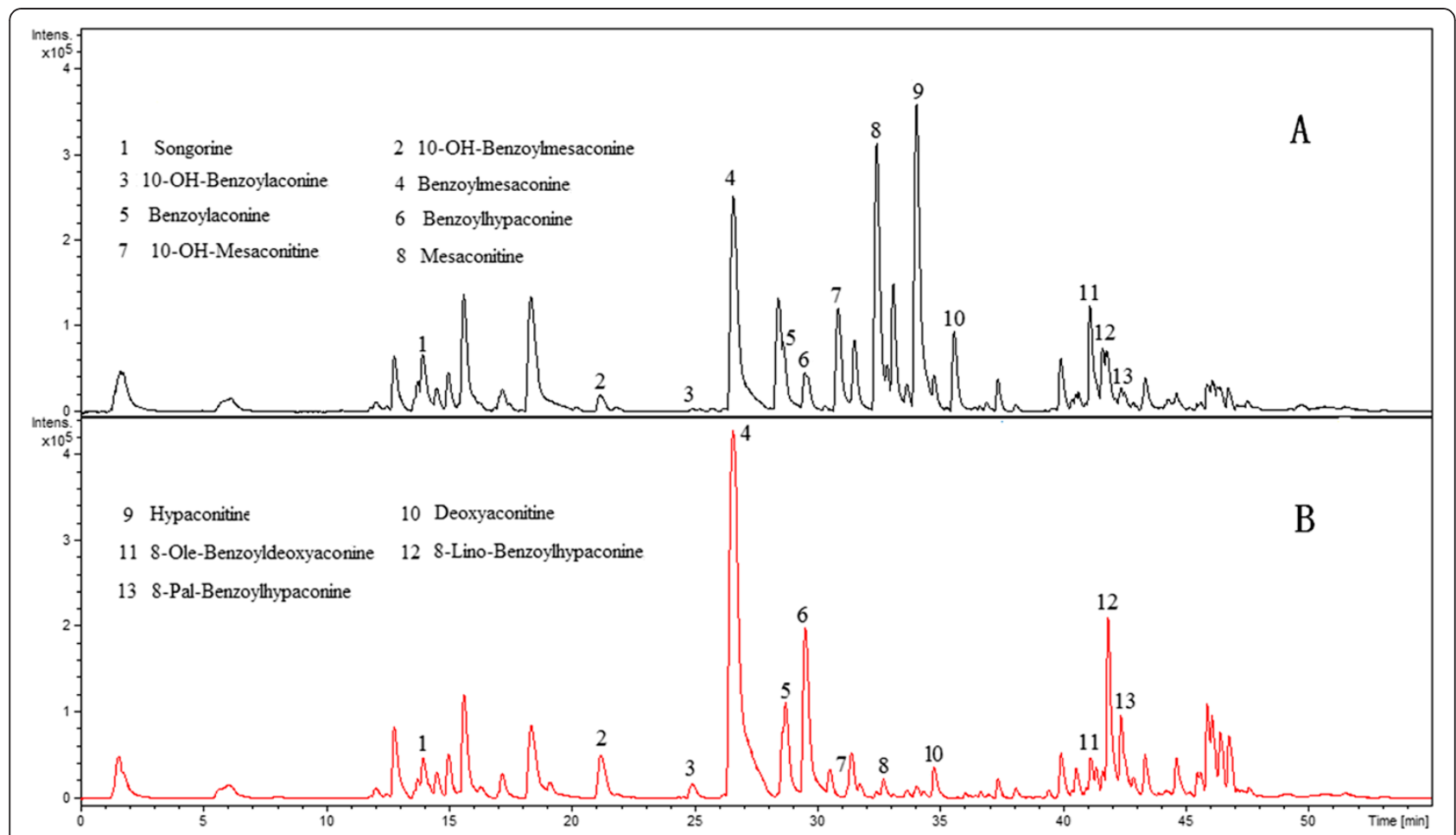

Figure 1 The representative base peak chromatograms of CW and ZCW by RPLC-Q-TOF/MS in the positive ESI mode. A, CW; B, ZCW.

Variables with a VIP value $>1$ have an above average influence on $\mathrm{Y}$ matrix explanation (classification). Therefore, metabolite ions with a VIP value $>1$ were set aside for further study. Twenty-two ions were identified in the positive mode. Then, the metabolite identification process was illustrated. We chose benzoylmesaconine (an ion at $\mathrm{m} / \mathrm{z} 590.3$ ) as an example to illustrate the biomarker identification process. MS/MS information of $572\left[\mathrm{M}+\mathrm{H}-\mathrm{H}_{2} \mathrm{O}\right]^{+}, 558\left[\mathrm{M}+\mathrm{H}-\mathrm{CH}_{4} \mathrm{O}\right]^{+}, 540[\mathrm{M}+\mathrm{H}-$ $\left.\mathrm{CH}_{6} \mathrm{O}_{2}\right]^{+}, 508\left[\mathrm{M}+\mathrm{H}-\mathrm{C}_{2} \mathrm{H}_{10} \mathrm{O}_{3}\right]^{+}, 105\left[\mathrm{M}+\mathrm{H}-\mathrm{C}_{24} \mathrm{H}_{39} \mathrm{NO}_{9}\right]^{+}$. The molecular formula of the benzoylmesaconine compound was determined as $\mathrm{C}_{31} \mathrm{H}_{43} \mathrm{NO}_{10}$. As mentioned above, 22 potential biomarkers between $\mathrm{CW}$ and ZCW were identified (Table 1). These potential biomarkers are: Dehydrated Benzoylmesaconine, Dehydrated Benzoylaconine, Benzoylaconine, Deoxyaconitine, 10-OH-Benzoylaconine, Hypaconitine, 10-OH-Aconitine, 10-OH-Benzoylmesaconine, Benzoyldeoxyaconine, Benzoylmesaconine, Deacetoxy 10-OH-Aconitine, 10$\mathrm{OH}-$ Mesaconitine, Mesaconitine, Benzoylhypaconine, 8-Pal-Benzoylhypaconine, 8-Linolen-Benzoylmesaconine, 8-Lino-Benzoylhypaconine, 14-Acetyl-Talatisamine, 8Pal-Benzoyldeoxyaconine, 8-Ole-Benzoylhypaconine, Songorine and 8-Ole-Benzoyldeoxyaconine.

\section{The processing mechanism of CW}

As we know, CW contains poisonous diester diterpene alkaloids (DDAs), less toxic monoester diterpene alkaloids
(MDAs) and amine diterpenoid alkaloids (ADAs) [2,4]. When $\mathrm{CW}$ is prepared to become $\mathrm{ZCW}$, the main components of DDA and MDA in CW was changed $[13,14]$. However, the specific change process and metabolite conversion mechanism are still unknown. Thus, we analysed change in metabolic markers to better understand the mechanism of attenuated toxicity. This understanding helps us more clearly explain the pao zhi process. Figure 4 shows that compared with CW, ZCW has lower DDAs concentrations (i.e., Mesaconitine, Deoxyaconitine and Hypaconitine), lower NEAs concentrations (i.e., 14-Acetyl-Talatisamine and Songorine), higher MDAs concentrations (i.e., 10-OH-Benzoylmesaconine, Benzoylmesaconine and Benzoylhypaconitine) and higher Lipo-Alkaloids concentrations (i.e., 8-Pal-Benzoylhypaconine, 8-Lino-Benzoylhypaconine and 8-Pal-Benzoyldeoxyaconine). Dehydrated Benzoylmesaconine, Dehydrated Benzoylaconine and Deacetoxy 10-OH-Aconitine are belonging to the intermediate product of DDAs converted into MDAs (IPDDAs). IPDDA content is lower in ZCW than in CW. The reason may be that the intermediate product is unstable and DDA content in ZCW is lower than that in $\mathrm{CW}$. The detoxification of $\mathrm{CW}$ is bound to a hydrolysis procedure. Samples of ZCW are thoroughly boiled with water or steamed for few hours. These results show that pao zhi can play a key role in detoxification. Information about the basic toxicity mechanism of $\mathrm{CW}$ and $\mathrm{ZCW}$ has also been provided. At present, metabolomics provides 


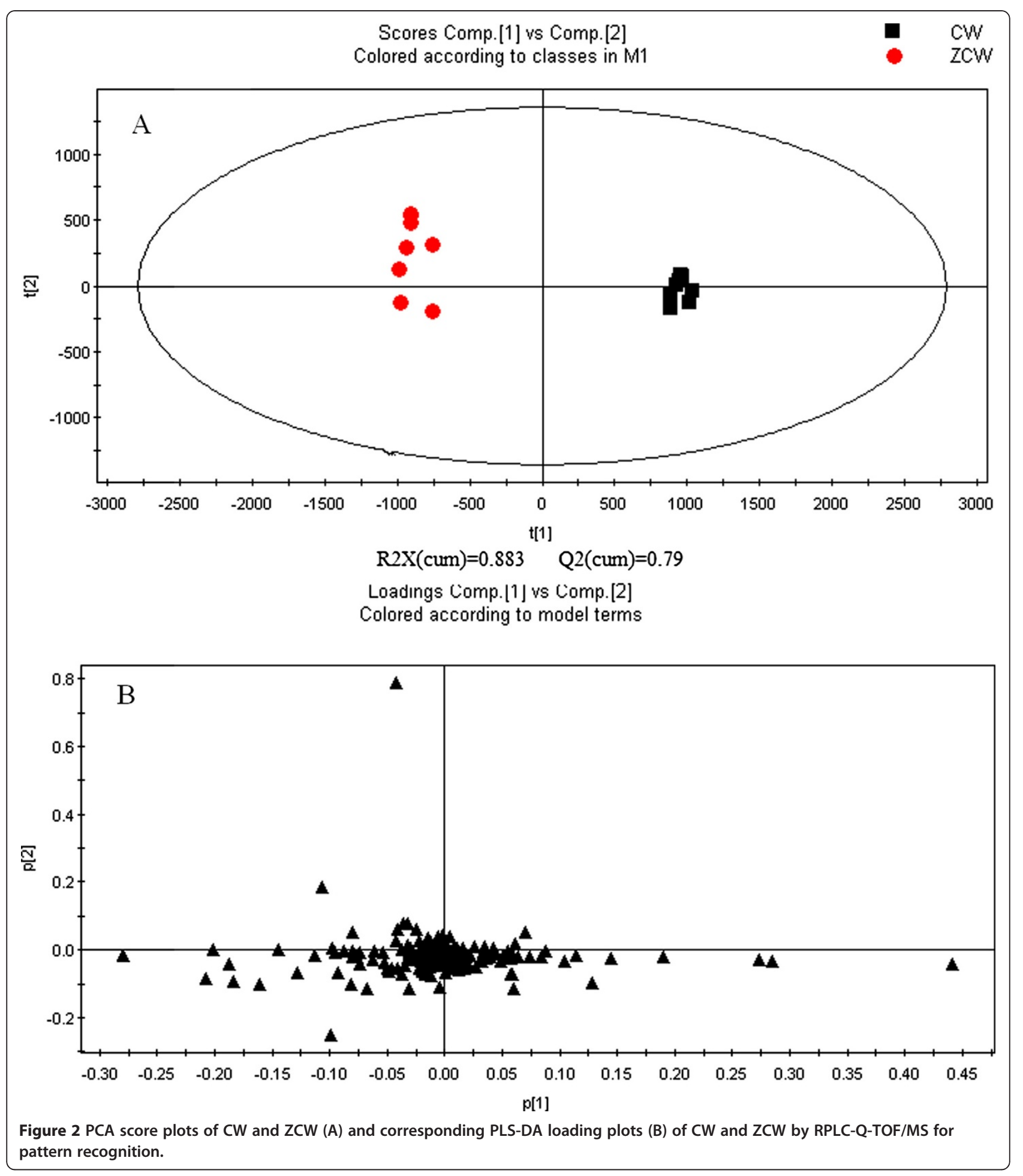

more useful information on the basic efficacy/toxicity mechanism of CW and its ZCW, as well as on potential metabolic biomarkers which can be used for the investigation of chemical transformation mechanisms underlying pao zhi.

\section{Experimental}

Chemicals, reference compounds and samples

Acetonitrile (ACN, HPLC-MS grade) from Merck (Darmstadt, Germany), formic acid (HPLC grade) from Sigma-Aldrich (Steinheim, Germany) and sodium formate 
Table 1 Identified 22 potential biomarkers between CW and ZCW by RPLC-Q-TOF/MS in positive ESI mode ${ }^{\text {a }}$

\begin{tabular}{|c|c|c|c|c|c|c|c|c|}
\hline \multirow[t]{2}{*}{ No } & \multirow{2}{*}{$\begin{array}{l}\mathrm{Rt} \\
(\min )\end{array}$} & \multicolumn{2}{|l|}{$\mathrm{m} / \mathrm{z}[\mathrm{M}+\mathrm{H}]^{+b}$} & \multirow[t]{2}{*}{ Identified compounds } & \multirow{2}{*}{$\begin{array}{l}\text { Molecular } \\
\text { formula }\end{array}$} & \multirow[t]{2}{*}{ MS/MS } & \multirow{2}{*}{$\begin{array}{l}\text { Structure } \\
\text { type }\end{array}$} & \multirow{2}{*}{$\begin{array}{l}\text { Content } \\
\text { variance }\end{array}$} \\
\hline & & Measured $\mathrm{m} / \mathrm{z}$ & Theoretical $\mathrm{m} / \mathrm{z}$ & & & & & \\
\hline \multirow[t]{5}{*}{1} & 30.80 & 648.3022 & 648.3015 & 10-OH-Mesaconitine & $\mathrm{C}_{33} \mathrm{H}_{45} \mathrm{NO}_{12}$ & $648[\mathrm{M}+\mathrm{H}]^{+}$ & & $\downarrow$ \\
\hline & & & & & & $556\left[\mathrm{M}+\mathrm{H}-\mathrm{C}_{3} \mathrm{H}_{8} \mathrm{O}_{3}\right]^{+}$ & & \\
\hline & & & & & & $538\left[\mathrm{M}+\mathrm{H}-\mathrm{C}_{3} \mathrm{H}_{10} \mathrm{O}_{4}\right]^{+}$ & & \\
\hline & & & & & & $528\left[\mathrm{M}+\mathrm{H}-\mathrm{C}_{4} \mathrm{H}_{8} \mathrm{O}_{4}\right]^{+}$ & & \\
\hline & & & & & & $105\left[\mathrm{M}+\mathrm{H}-\mathrm{C}_{26} \mathrm{H}_{41} \mathrm{NO}_{11}\right]^{+}$ & & \\
\hline \multirow[t]{5}{*}{2} & 32.36 & 632.3099 & 632.3065 & Mesaconitine & $\mathrm{C}_{33} \mathrm{H}_{45} \mathrm{NO}_{11}$ & $632[\mathrm{M}+\mathrm{H}]^{+}$ & & $\downarrow$ \\
\hline & & & & & & $572\left[\mathrm{M}+\mathrm{H}-\mathrm{C}_{2} \mathrm{H}_{4} \mathrm{O}_{2}\right]^{+}$ & & \\
\hline & & & & & & $540\left[\mathrm{M}+\mathrm{H}-\mathrm{C}_{3} \mathrm{H}_{8} \mathrm{O}_{3}\right]^{+}$ & & \\
\hline & & & & & & $512\left[\mathrm{M}+\mathrm{H}-\mathrm{C}_{4} \mathrm{H}_{8} \mathrm{O}_{4}\right]^{+}$ & & \\
\hline & & & & & & $105\left[\mathrm{M}+\mathrm{H}-\mathrm{C}_{26} \mathrm{H}_{41} \mathrm{NO}_{10}\right]^{+}$ & & \\
\hline \multirow[t]{5}{*}{3} & 32.77 & 662.3158 & 662.3171 & 10-OH-Aconitine & $\mathrm{C}_{34} \mathrm{H}_{47} \mathrm{NO}_{12}$ & $662[\mathrm{M}+\mathrm{H}]^{+}$ & & $\downarrow$ \\
\hline & & & & & & $602\left[\mathrm{M}+\mathrm{H}-\mathrm{C}_{2} \mathrm{H}_{4} \mathrm{O}_{2}\right]^{+}$ & & \\
\hline & & & & & & $570\left[\mathrm{M}+\mathrm{H}-\mathrm{C}_{3} \mathrm{H}_{8} \mathrm{O}_{3}\right]^{+}$ & DDAs & \\
\hline & & & & & & $542\left[\mathrm{M}+\mathrm{H}-\mathrm{C}_{4} \mathrm{H}_{8} \mathrm{O}_{4}\right]^{+}$ & & \\
\hline & & & & & & $105\left[\mathrm{M}+\mathrm{H}-\mathrm{C}_{27} \mathrm{H}_{43} \mathrm{NO}_{11}\right]^{+}$ & & \\
\hline \multirow[t]{5}{*}{4} & 33.97 & 616.3159 & 616.3116 & Hypaconitine & $\mathrm{C}_{33} \mathrm{H}_{45} \mathrm{NO}_{10}$ & $616[\mathrm{M}+\mathrm{H}]^{+}$ & & $\downarrow$ \\
\hline & & & & & & $584\left[\mathrm{M}+\mathrm{H}-\mathrm{CH}_{4} \mathrm{O}\right]^{+}$ & & \\
\hline & & & & & & $556\left[\mathrm{M}+\mathrm{H}-\mathrm{C}_{2} \mathrm{H}_{4} \mathrm{O}_{2}\right]^{+}$ & & \\
\hline & & & & & & $524\left[\mathrm{M}+\mathrm{H}-\mathrm{C}_{3} \mathrm{H}_{8} \mathrm{O}_{3}\right]^{+}$ & & \\
\hline & & & & & & $105\left[\mathrm{M}+\mathrm{H}-\mathrm{C}_{26} \mathrm{H}_{41} \mathrm{NO}_{9}\right]^{+}$ & & \\
\hline \multirow[t]{5}{*}{5} & 35.51 & 630.3271 & 630.3273 & Deoxyaconitine & $\mathrm{C}_{34} \mathrm{H}_{47} \mathrm{NO}_{10}$ & $630[\mathrm{M}+\mathrm{H}]^{+}$ & & $\downarrow$ \\
\hline & & & & & & $570\left[\mathrm{M}+\mathrm{H}-\mathrm{C}_{2} \mathrm{H}_{4} \mathrm{O}_{2}\right]^{+}$ & & \\
\hline & & & & & & $538\left[\mathrm{M}+\mathrm{H}-\mathrm{C}_{3} \mathrm{H}_{8} \mathrm{O}_{3}\right]^{+}$ & & \\
\hline & & & & & & $510\left[\mathrm{M}+\mathrm{H}-\mathrm{C}_{4} \mathrm{H}_{8} \mathrm{O}_{4}\right]^{+}$ & & \\
\hline & & & & & & $105\left[\mathrm{M}+\mathrm{H}-\mathrm{C}_{27} \mathrm{H}_{43} \mathrm{NO}_{9}\right]^{+}$ & & \\
\hline \multirow[t]{5}{*}{6} & 21.1 & 606.2881 & 606.2909 & 10-OH-Benzoylmesaconine & $\mathrm{C}_{31} \mathrm{H}_{43} \mathrm{NO}_{11}$ & $606[M+H]^{+}$ & & $\uparrow$ \\
\hline & & & & & & $574\left[\mathrm{M}+\mathrm{H}-\mathrm{CH}_{4} \mathrm{O}\right]^{+}$ & & \\
\hline & & & & & & $556\left[\mathrm{M}+\mathrm{H}-\mathrm{CH}_{6} \mathrm{O}_{2}\right]^{+}$ & & \\
\hline & & & & & & $524\left[\mathrm{M}+\mathrm{H}-\mathrm{C}_{2} \mathrm{H}_{10} \mathrm{O}_{3}\right]^{+}$ & & \\
\hline & & & & & & $105\left[\mathrm{M}+\mathrm{H}-\mathrm{C}_{25} \mathrm{H}_{43} \mathrm{NO}_{9}\right]^{+}$ & & \\
\hline \multirow[t]{5}{*}{7} & 24.87 & 620.3020 & 620.3065 & 10-OH-Benzoylaconine & $\mathrm{C}_{32} \mathrm{H}_{45} \mathrm{NO}_{11}$ & $620[\mathrm{M}+\mathrm{H}]^{+}$ & & $\uparrow$ \\
\hline & & & & & & $602[\mathrm{M}+\mathrm{H}-\mathrm{H} 2 \mathrm{O}]^{+}$ & & \\
\hline & & & & & & $570\left[\mathrm{M}+\mathrm{H}-\mathrm{CH}_{6} \mathrm{O}_{2}\right]^{+}$ & & \\
\hline & & & & & & $538\left[\mathrm{M}+\mathrm{H}-\mathrm{C}_{2} \mathrm{H}_{10} \mathrm{O}_{3}\right]^{+}$ & $M \cap A c$ & \\
\hline & & & & & & $105\left[\mathrm{M}+\mathrm{H}-\mathrm{C}_{25} \mathrm{H}_{45} \mathrm{NO}_{10}\right]^{+}$ & IVIDAS & \\
\hline \multirow[t]{5}{*}{8} & 26.53 & 590.2987 & 590.2960 & Benzoylmesaconine & $\mathrm{C}_{31} \mathrm{H}_{43} \mathrm{NO}_{10}$ & $590[\mathrm{M}+\mathrm{H}]^{+}$ & & $\uparrow$ \\
\hline & & & & & & $572\left[\mathrm{M}+\mathrm{H}-\mathrm{H}_{2} \mathrm{O}\right]^{+}$ & & \\
\hline & & & & & & $558\left[\mathrm{M}+\mathrm{H}-\mathrm{CH}_{4} \mathrm{O}\right]^{+}$ & & \\
\hline & & & & & & $540\left[\mathrm{M}+\mathrm{H}-\mathrm{CH}_{6} \mathrm{O}_{2}\right]^{+}$ & & \\
\hline & & & & & & $105\left[\mathrm{M}+\mathrm{H}-\mathrm{C}_{24} \mathrm{H}_{39} \mathrm{NO}_{9}\right]^{+}$ & & \\
\hline \multirow[t]{3}{*}{9} & 28.65 & 604.3098 & 604.3116 & Benzoylaconine & $\mathrm{C}_{32} \mathrm{H}_{45} \mathrm{NO}_{10}$ & $604[\mathrm{M}+\mathrm{H}]^{+}$ & & $\uparrow$ \\
\hline & & & & & & $586\left[\mathrm{M}+\mathrm{H}-\mathrm{H}_{2} \mathrm{O}\right]^{+}$ & & \\
\hline & & & & & & $572\left[\mathrm{M}+\mathrm{H}-\mathrm{CH}_{4} \mathrm{O}\right]^{+}$ & & \\
\hline
\end{tabular}




\begin{tabular}{|c|c|c|c|c|c|c|c|c|}
\hline & & & & & & $554\left[\mathrm{M}+\mathrm{H}-\mathrm{CH}_{6} \mathrm{O}_{2}\right]^{+}$ & & \\
\hline & & & & & & $105\left[\mathrm{M}+\mathrm{H}-\mathrm{C}_{25} \mathrm{H}_{41} \mathrm{NO}_{9}\right]^{+}$ & & \\
\hline \multirow[t]{4}{*}{10} & 29.46 & 574.3009 & 574.3011 & Benzoylhypaconine & $\mathrm{C}_{31} \mathrm{H}_{43} \mathrm{NO}_{9}$ & $574[\mathrm{M}+\mathrm{H}]^{+}$ & & $\uparrow$ \\
\hline & & & & & & $542\left[\mathrm{M}+\mathrm{H}-\mathrm{CH}_{4} \mathrm{O}\right]^{+}$ & & \\
\hline & & & & & & $510\left[\mathrm{M}+\mathrm{H}-\mathrm{C}_{2} \mathrm{H}_{8} \mathrm{O}_{2}\right]^{+}$ & & \\
\hline & & & & & & $105\left[\mathrm{M}+\mathrm{H}-\mathrm{C}_{24} \mathrm{H}_{39} \mathrm{NO}_{8}\right]^{+}$ & & \\
\hline \multirow[t]{4}{*}{11} & 34.58 & 602.3297 & 602.2960 & Deacetoxy 10-OH-Aconitine & $\mathrm{C}_{32} \mathrm{H}_{43} \mathrm{NO}_{10}$ & $602[\mathrm{M}+\mathrm{H}]^{+}$ & & $\downarrow$ \\
\hline & & & & & & $584\left[\mathrm{M}+\mathrm{H}-\mathrm{H}_{2} \mathrm{O}\right]^{+}$ & & \\
\hline & & & & & & $570\left[\mathrm{M}+\mathrm{H}-\mathrm{CH}_{4} \mathrm{O}\right]^{+}$ & & \\
\hline & & & & & & $552\left[\mathrm{M}+\mathrm{H}-\mathrm{CH}_{6} \mathrm{O}_{2}\right]^{+}$ & & \\
\hline \multirow[t]{4}{*}{12} & 28.38 & 572.2858 & 572.2854 & Dehydrated Benzoylmesaconine & $\mathrm{C}_{31} \mathrm{H}_{41} \mathrm{NO}_{9}$ & $572[\mathrm{M}+\mathrm{H}]^{+}$ & & $\downarrow$ \\
\hline & & & & & & $554\left[\mathrm{M}+\mathrm{H}-\mathrm{H}_{2} \mathrm{O}\right]^{+}$ & & \\
\hline & & & & & & $540\left[\mathrm{M}+\mathrm{H}-\mathrm{CH}_{4} \mathrm{O}\right]^{+}$ & & \\
\hline & & & & & & $522\left[\mathrm{M}+\mathrm{H}-\mathrm{CH}_{6} \mathrm{O}_{2}\right]^{+}$ & & \\
\hline \multirow[t]{3}{*}{13} & 29.56 & 586.3025 & 586.3011 & Dehydrated Benzoylaconine & $\mathrm{C}_{32} \mathrm{H}_{43} \mathrm{NO}_{9}$ & $586[\mathrm{M}+\mathrm{H}]^{+}$ & & $\downarrow$ \\
\hline & & & & & & $554\left[\mathrm{M}+\mathrm{H}-\mathrm{CH}_{4} \mathrm{O}\right]^{+}$ & & \\
\hline & & & & & & $536\left[\mathrm{M}+\mathrm{H}-\mathrm{CH}_{6} \mathrm{O}_{2}\right]^{+}$ & & \\
\hline \multirow[t]{3}{*}{14} & 32.52 & 570.3037 & 570.3061 & Benzoyldeoxyaconine & $\mathrm{C}_{32} \mathrm{H}_{43} \mathrm{NO}_{8}$ & $570[\mathrm{M}+\mathrm{H}]^{+}$ & & $\downarrow$ \\
\hline & & & & & & $552\left[\mathrm{M}+\mathrm{H}-\mathrm{H}_{2} \mathrm{O}\right]^{+}$ & & \\
\hline & & & & & & $520\left[\mathrm{M}+\mathrm{H}-\mathrm{CH}_{6} \mathrm{O}_{2}\right]^{+}$ & & \\
\hline \multirow[t]{2}{*}{15} & 40.58 & 850.5086 & 850.5100 & 8-Linolen-Benzoylmesaconine & $\mathrm{C}_{49} \mathrm{H}_{71} \mathrm{NO}_{11}$ & $850[\mathrm{M}+\mathrm{H}]^{+}$ & & $\downarrow$ \\
\hline & & & & & & $572\left[\mathrm{M}+\mathrm{H}-\mathrm{C}_{18} \mathrm{H}_{30} \mathrm{O}_{2}\right]^{+}$ & & \\
\hline \multirow[t]{2}{*}{16} & 41.05 & 852.5278 & 852.5620 & 8-Ole-Benzoyldeoxyaconine & $\mathrm{C}_{50} \mathrm{H}_{77} \mathrm{NO}_{10}$ & $852[\mathrm{M}+\mathrm{H}]^{+}$ & & $\downarrow$ \\
\hline & & & & & & $570\left[\mathrm{M}+\mathrm{H}-\mathrm{C}_{18} \mathrm{H}_{34} \mathrm{O}_{2}\right]^{+}$ & & \\
\hline \multirow[t]{2}{*}{17} & 41.77 & 836.5316 & 836.5307 & 8-Lino-Benzoylhypaconine & $\mathrm{C}_{49} \mathrm{H}_{73} \mathrm{NO}_{10}$ & $836[\mathrm{M}+\mathrm{H}]^{+}$ & & $\uparrow$ \\
\hline & & & & & & $556\left[\mathrm{M}+\mathrm{H}-\mathrm{C}_{18} \mathrm{H}_{32} \mathrm{O}_{2}\right]^{+}$ & LOAs & \\
\hline \multirow[t]{2}{*}{18} & 42.31 & 812.5295 & 812.5307 & 8-Pal-Benzoylhypaconine & $\mathrm{C}_{47} \mathrm{H}_{73} \mathrm{NO}_{10}$ & $812[\mathrm{M}+\mathrm{H}]^{+}$ & LUAS & $\uparrow$ \\
\hline & & & & & & $556\left[\mathrm{M}+\mathrm{H}-\mathrm{C}_{16} \mathrm{H}_{32} \mathrm{O}_{2}\right]^{+}$ & & \\
\hline \multirow[t]{2}{*}{19} & 42.45 & 838.5429 & 838.5464 & 8-Ole-Benzoylhypaconine & $\mathrm{C}_{49} \mathrm{H}_{75} \mathrm{NO}_{10}$ & $838[\mathrm{M}+\mathrm{H}]^{+}$ & & $\uparrow$ \\
\hline & & & & & & $556\left[\mathrm{M}+\mathrm{H}-\mathrm{C}_{18} \mathrm{H}_{34} \mathrm{O}_{2}\right]^{+}$ & & \\
\hline \multirow[t]{2}{*}{20} & 42.81 & 826.5433 & 826.5464 & 8-Pal-Benzoyldeoxyaconine & $\mathrm{C}_{48} \mathrm{H}_{75} \mathrm{NO}_{10}$ & $826[\mathrm{M}+\mathrm{H}]^{+}$ & & $\uparrow$ \\
\hline & & & & & & $570\left[\mathrm{M}+\mathrm{H}-\mathrm{C}_{16} \mathrm{H}_{32} \mathrm{O}_{2}\right]^{+}$ & & \\
\hline \multirow[t]{2}{*}{21} & 13.86 & 358.2358 & 358.2377 & Songorine & $\mathrm{C}_{22} \mathrm{H}_{31} \mathrm{NO}_{3}$ & $358[\mathrm{M}+\mathrm{H}]^{+}$ & & $\downarrow$ \\
\hline & & & & & & $340\left[\mathrm{M}+\mathrm{H}-\mathrm{H}_{2} \mathrm{O}\right]^{+}$ & NEAs & \\
\hline \multirow[t]{2}{*}{22} & 21.76 & 464.2977 & 464.3007 & 14-Acetyl-Talatisamine & $\mathrm{C}_{26} \mathrm{H}_{41} \mathrm{NO}_{6}$ & $464[\mathrm{M}+\mathrm{H}]^{+}$ & TVLAS & $\downarrow$ \\
\hline & & & & & & $446\left[\mathrm{M}+\mathrm{H}-\mathrm{H}_{2} \mathrm{O}\right]^{+}$ & & \\
\hline
\end{tabular}

${ }^{a}$ Note: $\uparrow$, content increased; $\downarrow$, content decreased. DDAs, diester diterpene alkaloids; MDAs, monoester diterpene alkaloids; NEAs, nonester alkaloids; LOAs, lipo-alkaloids. ${ }^{b}$ Note: all theoretical $\mathrm{m} / \mathrm{z}$ were calculated by Compass IsotopePattern of Bruke.

from Sigma-Aldrich (St. Louis, MO, USA) were purchased. Ultra-pure water was prepared using a Milli-Q SP system (Millipore, Bedford, MA, USA). Other solvents and chemicals were of analytical grade. CWs were collected from Jiangyou in Sichuan Province, which is the indigenous cultivating region for $\mathrm{CW}$. The identity of all CW samples (root and rhizome) was authenticated to be dried using morphological and histological methods by Dr. Lu Zhang. Preparation of processed CW was carried out according to Chinese Pharmacopoeia (CP) (2010). Voucher specimens of Aconitum carmichaeli Debx. and samples used in this study were deposited at Tianjin University of Traditional Chinese Medicine. Six reference compounds were purchased from the $\mathrm{Na}$ tional Institute for the Control of Pharmaceutical and Biological Products, China. 


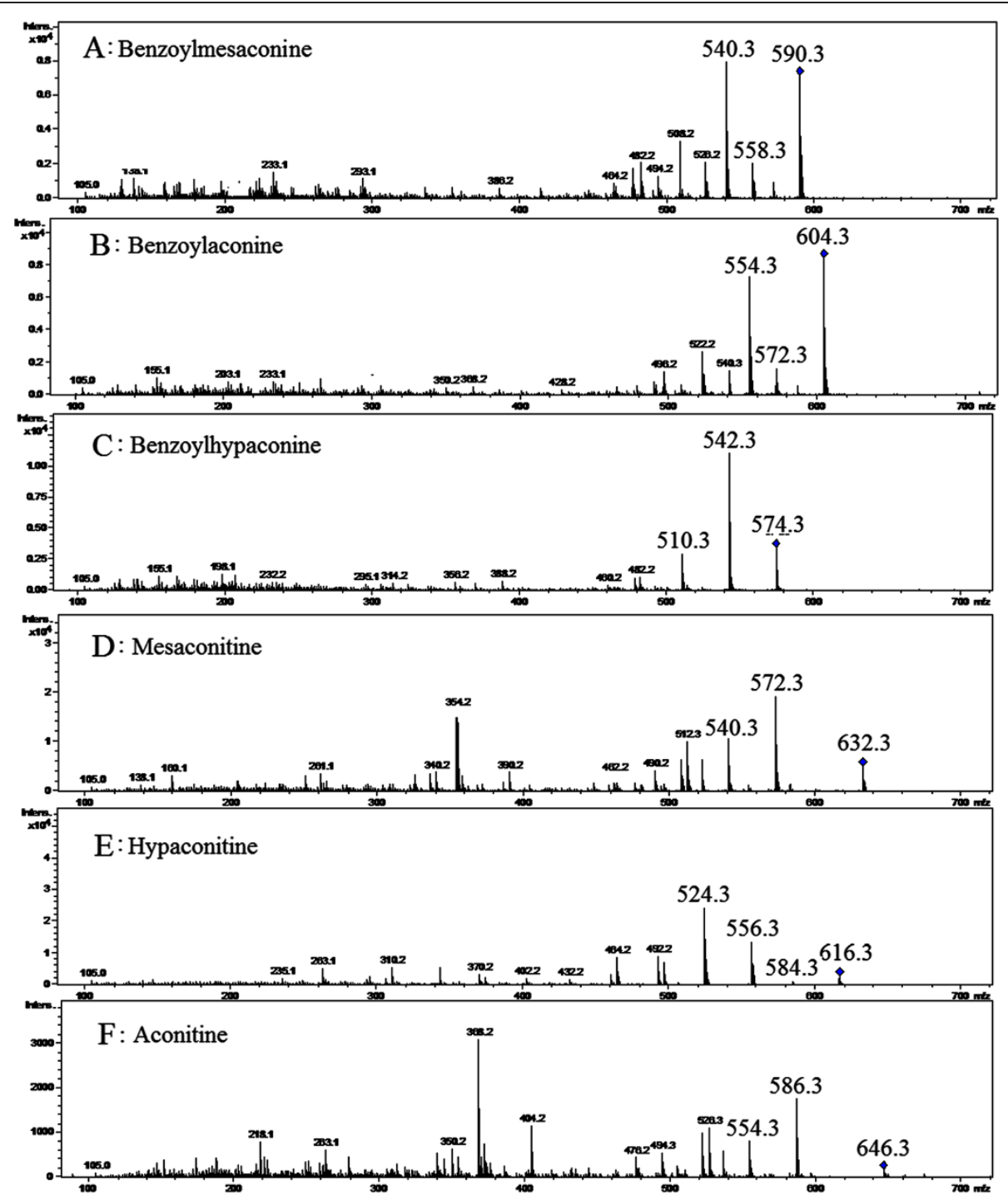

Figure $\mathbf{3}$ The $\mathbf{M S}^{2}$ spectra of six standard compounds. (A, Benzoylmesaconine; B, Benzoylaconine; C, Benzoylhypaconine; D, Mesaconitine; E, Hypaconitine; F, Aconitine).

\section{Liquid chromatography}

Liquid chromatography was performed with an Agilent 1200 system (Agilent Corp., MA, USA), equipped with a binary solvent delivery system and an autosampler. A mobile phase consisting of water (A) and acetonitrile (B) (each containing $0.1 \%$ formic acid) was used. In addition, separation was performed on an RP-C18 column (Agilent Zorbax SB-Aq, $2.1 \mathrm{~mm} \times 100 \mathrm{~mm}, 1.8 \mu \mathrm{m}$ particle size). RPLC elution condition was optimised as follows: $2 \%$ to $6 \% \mathrm{~B}$ (0 min to $5 \mathrm{~min}$ ), $6 \%$ to $13 \% \mathrm{~B}$ (5 $\mathrm{min}$ to $10 \mathrm{~min}$ ), $13 \%$ to $15 \%$ B (10 $\mathrm{min}$ to $15 \mathrm{~min}$ ), $15 \%$ to $20 \%$ B (15 min to $20 \mathrm{~min}$ ), $20 \%$ to $28 \% \mathrm{~B}$ ( $20 \mathrm{~min}$ to $25 \mathrm{~min}$ ), $28 \%$ to $40 \%$ B ( $25 \mathrm{~min}$ to $30 \mathrm{~min}), 40 \%$ to $85 \%$ B (30 $\mathrm{min}$ to $35 \mathrm{~min}$ ), $85 \% \mathrm{~B}$ (35 min to $40 \mathrm{~min}$ ), $85 \%$ to $2 \%$ B ( $40 \mathrm{~min}$ to $42 \mathrm{~min}$ ), isocratic at $2 \% \mathrm{~B}$ ( $42 \mathrm{~min}$ to $60 \mathrm{~min}$ ) and finally, washing and reconditioning of the column. Flow rate was set at $0.2 \mathrm{~mL} / \mathrm{min}$. The column and autosampler were maintained at $25^{\circ} \mathrm{C}$ and $10^{\circ} \mathrm{C}$, respectively. The injection volume of reference compounds and samples was $2 \mu \mathrm{L}$.

\section{Mass spectrometry}

Mass spectrometry analysis was carried out on a timeof-flight mass spectrometer Micro-TOF-QII (Bruker Daltonik $\mathrm{GmbH}$, Germany) using the following setting of tuning parameters: capillary voltage $4.5 \mathrm{kV}$, drying temperature $180^{\circ} \mathrm{C}$, nitrogen flow rate $6 \mathrm{~L} / \mathrm{min}$ and pressure 0.8 bar. The external calibration with sodium formate was clustered before individual measurements. Mass spectra were acquired in positive electrospray ionization (ESI) mode in a scan range from $100 \mathrm{~m} / \mathrm{z}$ to $1000 \mathrm{~m} / \mathrm{z}$ at a sampling rate of $2 \mathrm{~Hz}$. Reference 

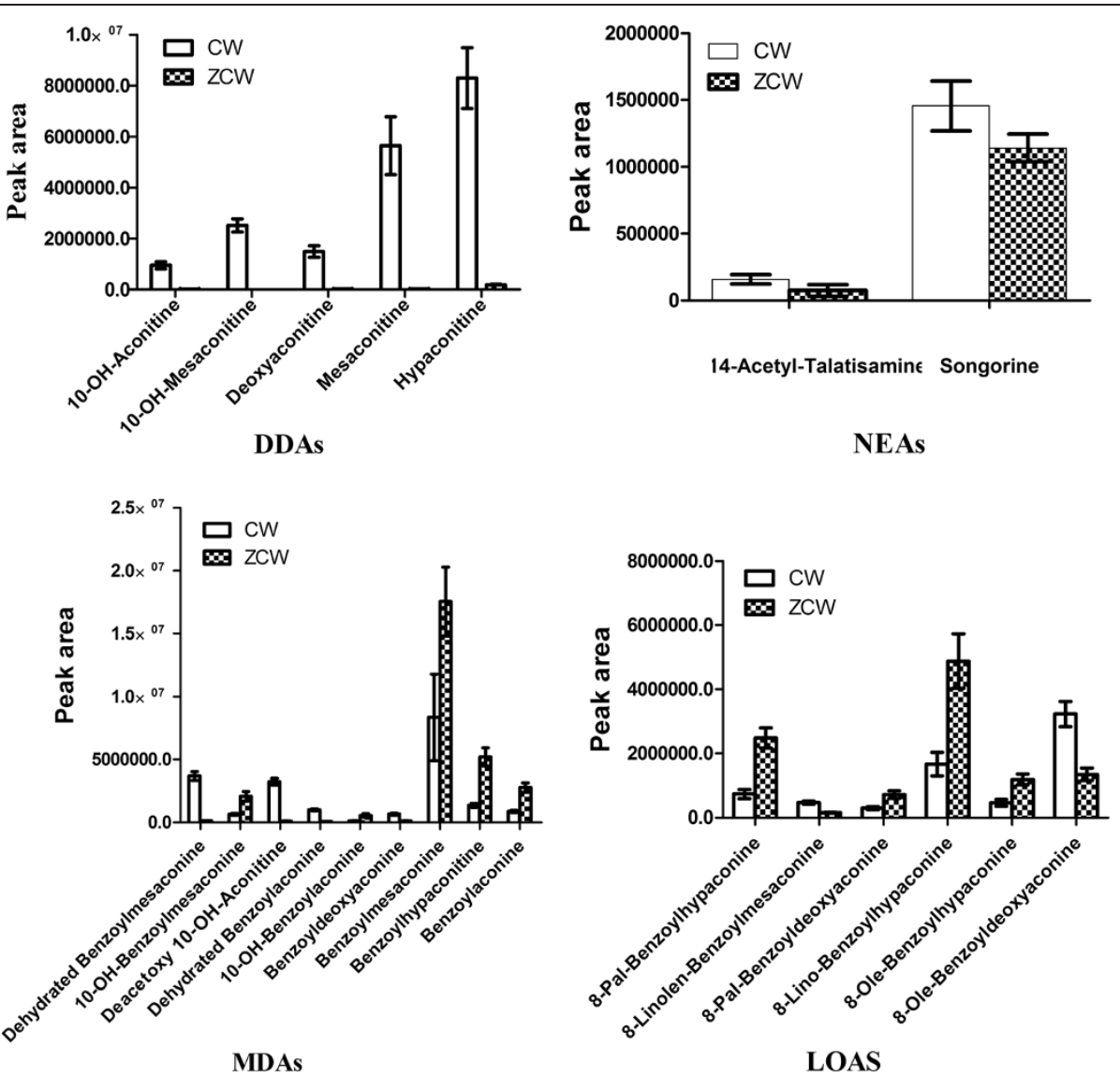

Figure 4 Graphical representation of 22 potential markers between $\mathrm{CW}$ and ZCW.

mass was scanned once every five scans for positive data collection.

\section{Sample preparation of $\mathrm{CW}$ and ZCW}

Eight samples of raw Aconitum carmichaeli Debx. were collected from Jiangyou, Sichuan Province. ZCW was obtained by boiling raw $\mathrm{CW}$ at $100^{\circ} \mathrm{C}$ for $8 \mathrm{~h}$, and then drying it according to CP (2010). The samples were pulverised, passed through a $0.30 \mathrm{~mm}$ sieve and stored in a desiccator.

Powder $(1.0 \mathrm{~g})$ was extracted with $70 \%$ ethanol $(10 \mathrm{~mL}$ and $8 \mathrm{~mL}$ ) by extracting it twice before being filtered and combined. The supernatant diluted to $100 \mathrm{~mL}$ with deionised water was then passed through a $0.22 \mathrm{~mm}$ filter. The filtrate was stored at $4^{\circ} \mathrm{C}$ in a refrigerator before being used for RPLC analysis.

\section{Data processing and pattern recognition analysis}

Raw data acquired from RPLC-Q-TOF/MS were pretreated using DataAnalysis 4.0 software (Bruker Daltonics) to find characteristic compounds with molecular features. Furthermore, mass data were exported to ProfileAnalysis 1.1 software (Bruker), which allowed for peak alignment, background noise subtraction and data reduction. Results provided a table of mass and retention time pairs with associated intensities for all detected peaks [15]. The main parameters were set as follows: retention time range $2 \mathrm{~min}$ to $55 \mathrm{~min}$, mass range 100 to 1000 , mass window 0.5 , retention time window $1 \mathrm{~min}$ and signal-to-noise $(\mathrm{S} / \mathrm{N})$ ratio threshold 5. Variables that did not exist in $80 \%$ of participants in one group were filtered [15]. To correct MS response shift during long analysis duration and different sample enrichment factors, data of each sample were normalised, thus ensuring that each sample was represented by a collection of variables to characterise its metabolic pattern before multivariate data analysis.

Normalised data were further exported to SIMCA-P 11.5 demo version software (Umetrics $A B$, Sweden) for multivariate data analysis [15]. Both PCA and PLS-DA were applied to investigate the metabolic profiles of the samples. PCA is an unsupervised data analysis technique that allows original data to be reduced to a few principal components whilst retaining features that mostly contribute to the variance [16]. By contrast, PLS-DA, is a supervised extension of PCA that uses class information to maximise separation among observation classes. 
Close sample clustering indicates their compositional similarity, whereas distant sample clustering suggests their diverse metabolomic compositions [17]. The significance of between-group differences for these metabolites was examined by the student's t-test using the computer software SPSS 13.0 (SPSS Inc., Chicago, USA). P-values less than 0.05 were selected to indicate statistical significance.

\section{Conclusions}

In this study, the comprehensive metabolomic characters of CW and ZCW by RPLC-Q-TOF/MS were investigated to guarantee clinical safety. Multivariate analyses successfully identified specific metabolite changes between CW and ZCW. In addition, 22 key biomarkers responsible for the detoxifying actions of pao zhi were discovered. The processing mechanism of CW were discussed according to the identified metabolites. This method is efficient, providing more accurate characterisations of traditional pao zhi detoxification.

\footnotetext{
Abbreviations

CW: Chuan Wu; TCM: Traditional Chinese medicine; ZCW: Prepared CW: HPLC: High-performance liquid chromatography; UV: Ultraviolet spectrophotometry; RPLC-Q-TOF/MS: Reversed phase liquid chromatography/quadrupole time-of-flight tandem mass spectrometry; PCA: Principal component analysis; PLS-DA: Partial least squares discriminant analysis; MS: Mass spectrometry; CP: Chinese pharmacopoeia;

ESI: Electrospray ionization; VIPs: Variable importance parameters; DDAs: Diester diterpene alkaloids; MDAs: Monoester diterpene alkaloids; ADAs: Amine diterpenoid alkaloids.
}

\section{Competing interests}

The authors declare that they have no competing interests.

\section{Authors' contributions}

All authors contributed to data analyses and drafting of the manuscript. All authors read and approve the final version.

\section{Acknowledgements}

This project was financially supported by the National Basic Research Program of China (973 Program) (2011CB505300, 2011CB505302) and the National Key Technology R\&D Program (No. 2011BAI07B08).

\section{Author details}

'Tianjin Key Laboratory of TCM Chemistry and Analysis, School of Traditional Chinese Materia Medica, Tianjin University of TCM, Tianjin 300193, P. R. China. ${ }^{2}$ Tianjin Huanhu Hospital, Tianjin 300060, P. R. China.

Received: 24 December 2012 Accepted: 15 February 2013 Published: 22 February 2013

\section{References}

1. Ameri A: The effects of Aconitum alkaloids on the central nervous system. Prog Neurobiol 1998, 56:211-235.

2. Singhuber J, Zhu M, Prinz S, Kopp B: Aconitum in traditional Chinese medicine: a valuable drug or an unpredictable risk? J Ethnopharmacol 2009, 126:18-30.

3. Bisset NG: Arrow poisons in China. Part II. Aconitum-botany, chemistry, and pharmacology. J Ethnopharmacol 1981, 4:247-336.

4. Sun H, Ni B, Zhang A, Wang M, Dong H, Wang X: Metabolomics study on Fuzi and its processed products using ultra-performance liquidchromatography/electrospray-ionization synapt high-definition mass spectrometry coupled with pattern recognition analysis. Analyst 2012, 137:170-185.
5. Cao G, Cai H, Zhang Y, Cong X, Zhang C, Cai B: Identification of metabolites of crude and processed Fructus Corni in rats by microdialysis sampling coupled with electrospray ionization linear quadrupole ion trap mass spectrometry. J Pharm Biomed Anal 2011, 56:118-125.

6. Li SL, Shen H, Zhu LY, Xu J, Jia XB, Zhang HM, Lin G, Cai H, Cai BC, Chen SL, Xu HX: Ultra-high-performance liquid chromatography-quadrupole/time of flight mass spectrometry based chemical profiling approach to rapidly reveal chemical transformation of sulfur-fumigated medicinal herbs, a case study on white ginseng. J Chromatogr A 2012, 1231:31-45.

7. Nicholson JK, Lindon JC, Holmes E: 'Metabonomics': understanding the metabolic responses of living systems to pathophysiological stimulivia multivariate statistical analysis of biological NMR spectroscopic data. Xenobiotica 1999, 29:1181-1189.

8. Dong H, Zhang AH, Sun H, Wang H, Lu X, Wang M, Ni B, Wang X: Ingenuity pathways analysis of urine metabolomics phenotypes toxicity of Chuanwu in Wistar rats by UPLC-Q-TOF-HDMS coupled with pattern recognition methods. Mol Biosyst 2012, 8:1206-1221.

9. Wang XJ, Wang HY, Zhang AH, Lu X, Sun H, Dong H, Wang P: Metabolomics study on the toxicity of aconite root and its processed products using ultra performance liquid-chromatography/electrosprayionization synapt high-definition mass spectrometry coupled with pattern recognition approach and ingenuity pathways analysis. J Proteome Res 2012, 11:1284-1301.

10. Zhu H, Wang C, Qi Y, Song F, Liu Z, Liu S: Fingerprint analysis of Radix Aconiti using ultra-performance liquid chromatography-electrospray ionization/tandem mass spectrometry (UPLC-ESI/MS(n)) combined with stoichiometry. Talanta 2013, 103:56-65.

11. Cui $P$, Han $H$, Wang $R$, Yang $L$ : Identification and determination of Aconitum alkaloids in Aconitum herbs and Xiaohuoluo pill using UPLCESI-MS. Molecules 2012, 17:10242-10257.

12. Sun A, Gao B, Ding X, Huang CM, But PP: Quantitative and qualitative analysis of aconitum alkaloids in raw and processed chuanwu and caowu by HPLC in combination with automated analytical system and ESI/MS/MS. J Anal Methods Chem 2012, 2012:936131.

13. Tan P, Liu Y, Guan J, Li F, Dong L, Qiao Y: Studies on new hydrolysate of aconitine using HPLC-MS(n) and quantum chemistry calculation. Chin J Chin Mater Med 2011, 36:2099-2101.

14. Huang QA, Zhang YM, He Y, Lu J, Lin RC: Studies on hydrolysis of aconitine. Chin J Chin Mater Med 2007, 32:2143-2145.

15. Lin L, Huang ZZ, Gao Y, Yan X, Xing J, Hang W: LC-MS based serum metabonomic analysis for renal cell carcinoma diagnosis, staging, and biomarker discovery. J Proteome Res 2011, 10:1396-1405.

16. Xu Y, Goodacre R: Multiblock principal component analysis: an efficient tool for analyzing metabolomics data which contain two influential factors. Metabolomics 2012, 8:s37-s51.

17. Szymańska E, Saccenti E, Smilde AK, Westerhuis JA: Double-check: validation of diagnostic statistics for PLS-DA models in metabolomics studies. Metabolomics 2012, 8:53-s16.

doi:10.1186/1752-153X-7-36

Cite this article as: Li et al:: Exploring potential chemical markers by metabolomics method for studying the processing mechanism of traditional Chinese medicine using RPLC-Q-TOF/MS: a case study of Radix Aconiti. Chemistry Central Journal 2013 7:36

\section{Publish with ChemistryCentral and every scientist can read your work free of charge \\ "Open access provides opportunities to our colleagues in other parts of the globe, by allowing anyone to view the content free of charge." \\ W. Jeffery Hurst, The Hershey Company.}

- available free of charge to the entire scientific community

- peer reviewed and published immediately upon acceptance

- cited in PubMed and archived on PubMed Central

- yours - you keep the copyright

Submit your manuscript here:

http://www.chemistrycentral.com/manuscript/<smiles>c1ccccc1</smiles>
Chemistry Central 